Man and Nature

L'homme et la nature

\title{
Figures et pouvoirs de l'abject : Le Neveu de Rameau ou les avatars de Narcisse
}

\section{Thierry Belleguic}

Volume 11, 1992

URI : https://id.erudit.org/iderudit/1012668ar

DOI : https://doi.org/10.7202/1012668ar

Aller au sommaire du numéro

Éditeur(s)

Canadian Society for Eighteenth-Century Studies / Société canadienne d'étude du dix-huitième siècle

ISSN

0824-3298 (imprimé)

1927-8810 (numérique)

Découvrir la revue

Citer cet article

Belleguic, T. (1992). Figures et pouvoirs de l'abject : Le Neveu de Rameau ou les avatars de Narcisse. Man and Nature / L'homme et la nature, 11, 1-23.

https://doi.org/10.7202/1012668ar

Copyright (C Canadian Society for Eighteenth-Century Studies / Sociéte canadienne d'étude du dix-huitième siècle, 1992
Ce document est protégé par la loi sur le droit d'auteur. L'utilisation des services d'Érudit (y compris la reproduction) est assujettie à sa politique d'utilisation que vous pouvez consulter en ligne.

https://apropos.erudit.org/fr/usagers/politique-dutilisation/ 


\section{Figures et pouvoirs de l'abject: Le Neveu de Rameau ou les avatars de Narcisse}

Narcisse, se penchant sur son image, découvre qu'elle n'est qu'un masque apposé sur son visage, qui se dérobe; pour accéder à soi, il devient Protée, être suspendu dans une forme, évanouie à sa saisie; et se rêve Argus, dont les yeux, semés dans l'univers, réfléchissent l'identité, multiple. ${ }^{1}$

\section{LE DIALOGISME DIDEROTIEN}

'Vertumnis, quotquot sunt, natus iniquis. ${ }^{2}$ Telle épigraphe dit la vérité du masque. Elle oppose à l'illusion de l'unicité identitaire du sujet la multiplicité protéiforme de Vertumne. Débarrassé de l'anamnèse platonicienne, ${ }^{3}$ l'espace ontologique du Neveu de Rameau se donne en effet à lire comme lieu d'inscription d'un sujet divisé, opaque, inconstant. Les contradictions, maintenues dans toute leur tension antithétique, y sont irréductibles à toute téléologie dialectique de la résolution. ${ }^{4}$ Dès lors, le sujet ne peut y être saisi que dans la mouvance des opérations phénoménologiques qui le constituent et le modifient constamment.

Par le parti pris d'ouverture dont elle procède, l'écriture diderotienne présente une des manifestations les plus symptomatiques de rupture de la structure monologique du discours philosophique traditionnel. ${ }^{5} \mathrm{Au}-$ delà du dialogue socratique et de sa réduction platonicienne, tous deux liés cependant par la prémisse consensuelle d'une vérité à atteindre, cette pratique scripturale entraîne le philosophe moraliste Moi et le bohème cynique Lui dans une dynamique polyphonique où Starobinski et Jauss ont su reconnaître un retour à la satire ménippée. ${ }^{6}$ Placée sous le signe de l'agonique, cette dynamique est structurée par deux isotopies opposées, celle de la fascination et celle de la répulsion, dans lesquelles les concepts d'abjection et de narcissisme tiennent une place centrale que nous nous proposons d'examiner.

Il s'agira, non seulement d'établir le rôle joué dans ce procès par les figures de l'abjection et du narcissisme, dont nous essaierons de 
montrer l'indissociable relation, mais également d'analyser l'articulation du discours dialogique sur une saisie du réel qui tente de penser l'hétérogène dans le mouvement labile de ses constantes transformations. Nous tenterons de montrer comment le discours de/sur l'abjection et sur le narcissisme à l'oeuvre dans Le Neveu de Rameau participe d'une entreprise de per/sub-version de la représentation classique -cartésienne- du sujet au dix-huitième siècle. A la suite de Galle et de Jauss, il s'agira par là même d'interpréter le travail plus général de Diderot de "'mise en dialogue" progressive de la doctrine des Lumières. ${ }^{7}$

\section{TOPOLOGIE ET ÉCONOMIE DE L'ABJECT}

Abject (latin abjectus, p.p. de abjicere):

Abjectus: 1) banal; 2) bas, humble; 3) abattu, sans courage.

Abjicere: I- (idée de séparation): 1) jeter loin de soi; 2) abandonner; II- (idée d'abaissement): 1) jeter en bas, jeter à terre; 2) abattre, terrasser; 3 ) abattre au sens moral.

L'abjection est mouvement (jacere: jeter); elle procède de la violence et présuppose une im/ex/pulsion initiale. L'abject dit ainsi le rapport, ou plutôt la tension entre un intérieur et un extérieur, entre soi et non-soi, c'est-à-dire entre soi et l'autre, ou encore entre soi et soi, c'est-à-dire entre soi et l'autre-au-dedans-de-soi. ${ }^{8}$ Ajoutons à cela que l'expresse désignation du bas et de la chute, outre le motif général de l'avilissement, peut être lue dans le cadre du Neveu de Rameau comme le signe d'un avatar de la tradition carnavalesque.

\section{L'ABJECTION DIDEROTIENNE: ENTRE CLASSICISME ET MODERNITÉ}

Au XIVème siècle, le terme 'abjection' n'est utilisé que dans le champ du religieux, où il signifie l'humilité profonde du croyant devant Dieu, la négation de sa propre identité, la reconnaissance de son état de déréliction et de déchéance depuis la chute, indiquant par là même une scission originelle du sujet, un manque-à-être irrémédiable. C'est donc bel et bien son statut d'expulsé, en l'occurrence ici du jardin d'Eden, qui rend le sujet abject. 
$\mathrm{Au}$ XVIIIème siècle, 'abjection' désigne la basse condition d'une personne, par conséquent l'état servile où la maintient cette condition et, par extension, le mépris où cette servilité la tient. Le terme, s'il présuppose un rapport à l'éthos, au sens aristotélicien de caractère, d'habitus, n'en ressortit pas moins, prioritairement, au politique et à l'économique. ${ }^{10}$ Dans le prolongement de ce passage déjà remarquable du spirituel à l'économique et au politique, en lui-même symptomatique de la transformation de la doxa, notre analyse aura pour propos de désigner dans Le Neveu de Rameau des points de résistance où se donnent à lire les signes latents de l'évolution du champ sémantique de ce terme. L'émergence d'un sujet se détachant du collectif, et qui plus est divisé, offre en effet la possibilité d'une analyse de l'exploration diderotienne de la psyché, entendue -dans une acception qui, dépassant l'opposition platonicienne au soma, inclut ce dernier, comme ensemble des aspects du comportement individuel, c' est-à-dire comme Ego.

L'abjection du Neveu s'inscrit dans la tradition carnavalesque qui oppose le maître à l'esclave, le roi à son fou, le philosophe au morosophe. ${ }^{11}$ Cependant, alors que le carnaval est un renversement, un rite cathartique qui, en ultime ressort, confirme le fondement de la Loi dans sa légitimité, Le Neveu de Rameau participe d'une pratique scripturale qui dénie, par ses apories, toute positivité heuristique au dialogue. Le texte diderotien marque l'exhaussement de l'intérêt particulier par rapport à l'intérêt général, la négation, non pas de la Loi, mais de toute loi, et signe ainsi l'émergence d'une conscience de soi exacerbée en proie à la fragmentation. En termes bakhtiniens, Le Neveu de Rameau se situe entre l'universalité de la folie joyeuse du grotesque populaire, dont il conserve la bouffonnerie, et la sombre et tragique folie du grotesque romantique, dont il annonce l'irrémédiable solipsisme. ${ }^{12}$

Le Neveu de Rameau constitue donc un véritable tournant dans l'histoire du dialogue saturnal, en ce que s'y dessinent à la fois l'intégration d'une tradition, sa désignation dans le texte même, que ce soit par l'épigraphe horacienne ou les références rabelaisiennes, et la subversion de cette même tradition. ${ }^{13}$ Il ne s'agit cependant pas de faire de Diderot un Artaud ou encore un Céline, mais de souligner la dimension essentielle que revêt l'abject dans l'élaboration du sujet diderotien, tant dans la saisie politique des rapports entre le collectif (doxa) et le particulier (para-doxa), dans la saisie sociale des rapports interindividuels (soi et l'autre) que dans la saisie psychique des rapports entre soi et l'autre-audedans-de-soi.

C'est du Neveu de Rameau comme inscription d'un glissement du sens de l'abject que notre titre veut rendre compte. 'Figures et pouvoirs de l'abject: Le Neveu de Rameau ou les avatars de Narcisse': l'on aura bien 
sûr deviné une ouverture sur Pouvoirs de l'Horreur, où Julia Kristeva analyse l'abjection comme point nodal du processus de constitution du sujet. La démarche de Kristeva n'est pertinente à notre propos que dans la dimension en quelque sorte phénoménologique de sa première partie, intitulée 'Approche de l'abjection,' où elle articule le narcissique et l'abject dans le même mouvement définitoire et fait de l'abject une pré-condition du narcissique. $^{14}$

\section{ABJECTION ET ONTOLOGIE}

Par sa problématisation du dualisme fondateur de la métaphysique cartésienne, Le Neveu de Rameau rend compte d'un tournant décisif dans l'histoire de la représentation du sujet. Telle saisie atomiste de l'univers annihile dans un mouvement tout épicurien la suprématie de la ratio. ${ }^{15}$ Le champ définitoire ainsi circonscrit consacre l'irréductible opacité du sujet moderne, ainsi que l'inéluctabilité de son inachèvement dans une solitude ontologique que Julia Kristeva évoque dans Histoires d'Amour en ces termes:

[...] Narcisse découvre dans la douleur et la mort l'aliénation constitutive de son image propre. Privé de l'Un, il n'a pas de Salut; l'altérité s'est ouverte en lui-même. Il n'a plus le noûs pensant de l'Antiquité pour aborder l'autre comme une pluralité [...] Il n'a pas le noûs aimant plotinien pour pouvoir échapper à son altérité dans la fusion avec l'Un. S'il est seul à seul, son altérité ne se boucle pas en totalité, elle ne devient pas intériorité. Elle reste ouverte, béante, mortelle, parce que privée $d^{\prime} U{ }^{16}$

Dans cette perspective, il est possible de lire Le Neveu de Rameau dans le sens d'une tentative de réflexion sur un sujet divisé, en instance de dispersion, voire absent à lui-même. L'on sait par ailleurs toute la fascination de Diderot pour l'exclusion, la marginalité et la monstruosité, c'est-à-dire pour une pensée de l'écart aux vertus centrifuges.

S'appuyant sur une isotopie de l'abjection recouvrant, entre autres et sans ici analyser le contexte précis de leurs occurrences, les termes 'abject,' 'abjection,' 's'avilir,' 'avilissement,' 'bassesse,' 'dépravation,' 'ignominie,' 'infâme,' 'infamie,' 'perversité,' 'vaurien,' 'vice,' 'vicieux,' 'vil(e/es),' 'vilenie, ${ }^{17}$ notre analyse des rapports entre Moi et Lui porte sur l'économie de concomitance et d'échange dans laquelle l'abjection semble s'organiser à partir d'un va-et-vient constant entre introspection et projection. En d'autres termes, et dans le cadre dialogique du texte 
diderotien, il s'agit de considérer un double mouvement. Dans le premier, Lui joue le rôle de catalyseur de la prise de conscience par Moi de sa propre abjection. L'abjection de l'autre, toujours-déjà inscrite en soi, y joue le rôle de miroir, et Moi le philosophe devient alors le siège d'affects antagonistes. (Moi doit être entendu ici à la fois comme sujet psychique et comme 'symbole' de la doxa). Dans le second mouvement, qui doit être également évalué dans la double dimension mentionnée, Lui affirme son identité/originalité en revendiquant son abjection.

\section{ABJECTION, IDENTITÉ ET THÉORIE DU STERCORAIRE}

Dejicere: 1) jeter à bas, précipiter. 2) terme de médecine: évacuer, faire évacuer; relâcher le ventre; vomir. 3) terme militaire: déloger l'ennemi. 4) terme de droit: chasser d'une propriété, réellement et non pas symboliquement. 5) terme de marine: être entraîné loin de sa route. 6) abaisser au sens figuré.

Dejectus: I- 1) part. de dejicere; 2) (adj.) bas, en contrebas; 3) abattu; II- action de jeter à bas.

Dejectibilis: digne d'être rejeté, méprisable.

L'ab-jection et la dé-jection structurent le même champ sémantique de détachement, d'expulsion et de chute. En effet, l'abjectio et le dejectio participent tous deux du même mouvement de déperdition identitaire puisque le sujet se voit méprisé, rejeté dans l'indistinction du commun, dans l'indifférenciation du général.

Or, une réflexion de Lui doit tout particulièrement requérir notre attention, qui unit déjection et abjection dans une approbation de soi toute hédoniste: 'Le point important est d'aller aisément, librement, agréablement, copieusement tous les soirs à la garde-robe, o stercus pretiosum!' (25). 'O précieux excrément!': le credo du Neveu va bien au-delà d'une simple valorisation des fonctions corporelles et des plaisirs qu'elles peuvent nous offrir; au-delà même de l'acceptation du corps, et de son intégration comme élément constitutif à part entière du procès identitaire, il s'agit d'inverser le rapport à l'abject - au sens strict de 'ce que l'on expulse de soi': le dégoût se transforme en plaisir, la honte en revendication narcissique. Le stercoraire joue donc ici un rôle prépondérant dans l'expression identitaire et participe d'une théorie énoncée comme suit par Michel Serres dans Le Parasite: 
[...] Qu'est-ce donc que le propre? C'est ce qui n'est pas sale. Qu'est-ce qui n'est pas sale? Mon propre, justement. Stercus suum bene olet: voici le fondement de la propriété, que sa propre fiente sent bon [...] le propre, c'est le propre, et la propriété n'est que la propreté. Cette chose horrible pour vous, c'est la mienne en tant que je suis seul à n'en pas concevoir exécration ni répugnance. [...] Voilà une théorie stercoraire. Elle fournit un fondement, si j'ose dire, à la propriété privée. $^{18}$

i.e. aussi à la constitution de l'identité de soi.

\section{ABJECTION ET PARASITISME}

Le Neveu de Rameau est un parasite, pique-assiette insatiable à la solde du plus offrant. L'étymologie du terme 'parasite' nous apprend par ailleurs que si l'acception la plus courante en grec classique de sitos est 'nourriture,' elle est parfois 'excrément'. Le para-site Rameau n'est donc plus seulement celui qui mange 'à côté' de son hôte Bertin, il est aussi celui qui vit à proximité ou qui entretient une relation particulièrement étroite avec le stercoraire, avec la déjection, et donc avec l'abjection. L'abject étant ce que l'on expulse de soi et qui dès lors provoque notre dégoût par le statut même d'exclusion dont il est frappé, ${ }^{19} 1^{\prime}$ 'originalité du Neveu consiste, comme nous l'avons mentionné, à renverser la proposition qui associe l'abjection au mépris et à la perte identitaire, en faisant de l'approbation ${ }^{20}$ de l'abjection un élément du procès de constitution et d'affirmation de soi. Encore convient-il de rappeler que le schème d'exclusion fonctionne tant au niveau du politique, du social et du collectif, la société civile saisie comme tout organique excluant les parasites et marginaux dans le mouvement qui la constitue, qu'au niveau de l'(inter)individuel et du 'psychologique', le sujet se définissant et se constituant par rapport à l'autre.

L'abjection serait donc le fondement de la 'propriété de soi,' c'est-àdire de l'identité, que ce soit celle d'une nation, d'un groupe social ou d'un individu. Dans le cas du rapport entre Moi et Lui, ce processus correspond à un double mouvement: celui par lequel la réaction de dégoût du philosophe face à l'abjection du Neveu désigne à ce dernier ce qui est exclusivement et irréductiblement sien, et celui par lequel la reconnaissance et la revendication de cette irréductiblilité fonde l'identité même du Neveu. Il va sans dire que ce que le sujet projette comme étant l'abjection de l'autre est dans bien des cas la sienne propre. Tel est en effet le sens de notre analyse des rapports entre Lui et Moi. 
L'abject dépasse ainsi la seule relation à la saleté (champ du physiologique), à la bassesse de condition (champ du social), ou à l'exclusion du collectif (champ du politique), pour circonscrire une problématique plus générale: ce qui rend abject est le signe d'une fonction fondamentale qui est de l'ordre de la perturbation:

Ce n'est donc pas l'absence de propreté ou de santé qui rend abject, mais ce qui perturbe une identité, un système, un ordre. Ce qui ne respecte pas les limites, les places, les règles. L'entre-deux, l'ambigu, le mixte. ${ }^{21}$

Nous avons développé ailleurs l'idée selon laquelle l'isotopie parasitaire à l'oeuvre dans Le Neveu de Rameau, tant au niveau biologique, social que cybernétique, participe d'une pensée de l'hétérogène qui fait du parasite l'être même de la relation. ${ }^{22}$ La figure du Neveu y apparaît de façon privilégiée comme la représentation du mixte, de l'hybride, de l'impur. Le Neveu dit l'instabilité de l'identité, la mouvance des déterminations, la perméabilité des définitions. Il dit aussi qu'il ne saurait y avoir $\mathrm{d}^{\prime}$ identité, quelle qu'elle soit, sans abjection. L'abjection serait ainsi l'être de la relation identitaire.

Au sein de cet espace, où l'organique, l'économique et le cybernétique sont isotopiques, l'abject tient une place prépondérante. Pris dans un processus organico-économique de production, de distribution, de consommation et de transformation, le manger, l'in-gere, est indissociable du dé-jecter, de l'exi-gere. La parole, qui est de l'ordre de la communication, mais aussi de l'ordre du physiologique, devient chez le Neveu le pendant de l'alimentaire et, à l'autre bout de la chaîne de transformation, du stercoraire:

Il s'était introduit, je ne sais comment, dans quelques maisons honnêtes, où il avait son couvert, mais à la condition qu'il ne parlerait pas, sans en avoir obtenu la permission. Il se taisait, et mangeait de rage. Il était excellent à voir dans cette contrainte. S'il lui prenait envie de manquer au traité, et qu'il ouvrît la bouche; au premier mot, tous les convives s'écriaient, $O$ Rameau. Alors la fureur étincelait dans ses yeux, et il se remettait à manger avec plus de rage. (6)

Soumis à la tyrannie du physiologique, le Neveu ne peut que se remplir ou se vider. Sa propension à parler, associée au plaisir anal de l'aveu, est une véritable logorrhée: 'Est-ce qu'il [Bertin] ne sait pas que je suis comme les enfants, s'exclame-t-il, et qu'il y a des circonstances où je laisse tout aller sous moi?' (64-65) 
Le constat d'abjection du parasite s'impose presque de lui-même: le parasite perturbe l'ordre; or, l'abject est 'ce qui perturbe une identité, un système, un ordre'; donc le parasite est abject. Lui est un parasite, donc Lui est abject. N'est-il d'ailleurs pas expulsé de la maison Bertin?

\section{ABJECTION ET AGONIQUE}

Le mode de relation qui régit les rapports entre Lui et Moi, en ce qu'il ressortit au dialogique, est fondamentalement agonique. Dans ce cadre agonique, et dans la tradition carnavalesque précédemment mentionnée, la logorrhée dont est frappé Lui ne contribue-t-elle pas à conchier son interlocuteur le philosophe? L'analyse des registres respectifs de Moi et de Lui, tant lexicaux que thématiques, met en évidence un phénomène de contamination, parfois réciproque, des discours de l'un par l'autre. ${ }^{23}$ Un lieu de passage s'y voit désigné, où soi est investi par l'autre, car il ne s'agit pas tant d'échange que de lutte, de passage forcé. Dans cette confrontation, c'est Moi le philosophe qui paraît le plus menacé, en ce que lui seul présuppose fixité, homogénéité et préséance de la ratio.

La modalisation de la voix narrative de Moi révèle la coexistence de deux registres antithétiques, celui de l'attraction et celui de la répulsion, celui de la fascination et celui du mépris, signalant par là même le surgissement du mixte et de l'impur dans l'espace 'homogène' du philosophe. La pantomime du proxénète et de la jeune fille, au cours de laquelle Lui donne sa bassesse en spectacle, produit symptomatiquement sur le philosophe des effets contradictoires dont l'impossible résolution provoque une réaction somatique de douleur:

Je l'écoutais, et à mesure qu'il faisait la scène du proxénète et de la jeune fille qu'il séduisait, l'âme agitée de deux mouvements opposés, je ne savais si je m'abandonnerais à l'envie de rire, ou au transport de l'indignation. Je souffrais. [...] J'étais confondu de tant de sagacité et de tant de bassesse; d'idées si justes et alternativement si fausses; $d$ 'une perversité si générale de sentiments, d'une turpitude si complète, et d'une franchise si peu commune. Il s'aperçut du conflit qui se passait en moi .... (24; nous soulignons)

Et le philosophe de répondre au Neveu, qui s'enquiert des raisons du trouble qu'il lui voit: 'Ah, malheureux, dans quel état d'abjection, vous êtes né ou tombé' (25). 


\section{ABJECTION ET PERVERSITE}

Comme le note Moi le philosophe, Lui n'est pas seulement abject, il est également pervers. Est pervers, étymologiquement, ce qui tourne dans une direction opposée aux dieux, garants de l'ordre, de la doxa. Pervertir (pervertere) signifie littéralement 'renverser', 'retourner'. Lui est pervers parce qu'il renverse l'ordre général, le perturbe: ${ }^{24}$ 'Mais ne voyez-vous pas, s'exclame Moi, qu'avec un pareil raisonnement vous renversez l'ordre général [...]?' (14).

Lui est pervers parce qu'il affirme la chose et son contraire; il déclare: 'je suis conséquent' (11), puis: 'moi, je suis sans conséquence' (18). Il est à la fois conséquent et inconséquent. Il est à la fois sujet autonome qui se désigne dans une transparence toute stratégique et sujet fissuré, opaque, insaisissable.

Lui est pervers en ce sens qu'il est double: il est à la fois la loi et sa transgression. Il est à la fois en dedans et en dehors du système économique et social d'échanges. Son attitude soumise n'est qu'une façade: il mime avec beaucoup d'orthodoxie le cérémonial parasitaire. Il prend des poses: il parodie. Il ne se conforme que par pur intérêt égoïste, égoïsme dont Bentham dit à la même époque qu'il peut être un altruisme s'il obéit au principe utilitaire d'un monde du plus grand bonheur pour le plus grand nombre, où la vertu est ce qui maximise les plaisirs et minimise les peines. L'on conçoit toute la distance qui sépare la transparence utopique du totalitaire panoptikon de l'opacité du Neveu de Rameau, qui consacre la primauté du sujet sur la collectivité. La perversité serait alors chez le Neveu le signe et la mise en application stratégique de la prise de conscience approbatrice de l'abjection de $s o i{ }^{25}$

\section{JEAN-FRANÇOIS LE FATALISTE}

L'absence totale de sentiment de culpabilité face à son égoïsme et à sa perversité repose chez le Neveu sur une théorie du déterminisme d'une nature aveugle et contingente. Il en tire des conséquences, pédagogiquement subversives, qui rappellent le Diderot critique d'Helvétius:

S'il [son fils] est destiné à devenir un homme de bien, je n'y nuirai pas. Mais si la molécule voulait qu'il fût un vaurien comme son père, les peines que j'aurais prises pour en faire un homme honnête lui seraient très nuisibles: l'éducation croisant sans cesse la pente de la molécule, il serait tiré comme par deux forces contraires et marcherait tout de guingois dans le chemin de la vie .... $(90)^{26}$ 
Le déterminisme matérialiste de Diderot subordonne la liberté à l'affirmation, paradoxale, de la nécessité pour le sujet de s'émanciper d'un libre-arbitre illusoire. La démarche est de conséquence. Ainsi le Neveu suit-il la pente de sa nature puisque, dit-il, 'je puis faire mon bonheur par des vices qui me sont naturels' (44). Quel est donc son plaisir? 'Se moquer en dedans de la bêtise de ceux qu'on enivre' (51), 'rire en dessous de l'impertinent qu'on admir[e]' (53), où l'on voit s'affirmer une pensée du masque et du simulacre qui n'expose sa bassesse que pour mieux pervertir:

L'abject, écrit kristeva, est pervers car il n'abandonne ni n'assume un interdit, une règle ou une loi; mais les détourne, fourvoie, corrompt; s'en sert, en use, pour mieux les dénier [...] c'est le cynique [...]; il rassoit son pouvoir narcissique en feignant d'exposer ses abîmes [...] La corruption est sa figure la plus répandue, la plus évidente. Elle est la figure socialisée de l'abject. ${ }^{27}$

N'est-ce pas là le portrait même de la duplicité du Neveu, personnage corrompu qui exhibe son abjection dans une apparente franchise qui n'est, en tout état de cause, qu'un autre travestissement? L'on sait par ailleurs qu'il donnerait un de ses doigts pour avoir trouvé le masque (52).

\section{ENTRE DIOGÈNE ET PHRYNÉ}

Selon ses propres termes, Lui se situe 'entre Diogène et Phryné' (8), entre le cynique et la courtisane. L'évocation de la catin Phryné, choisie par le sculpteur Praxitèle pour servir de modèle au modèle idéal et absolu de la beauté qu'est Aphrodite, est emblématique. A la diade classique du Bon et du Beau, elle vient perversement superposer celle du Vil et du Sublime. Par ailleurs, Phryné s'exhibant pour désarmer le jugement de l'Aréopage n'est pas sans rappeler le Neveu exhibant son avilissement afin de persuader le philosophe: au-delà d'évidentes différences se dessine un schème général de séduction procèdant par dévoilement narcissique de soi.

La revendication d'une filiation diogénique, quant à elle, convoque la figure du paradoxe. En transgressant les interdits, en désobéissant à la loi, aux règles, en pervertissant les normes, en sapant les fondements de la coutume et de l'usage, le cynisme de Diogène de Sinope vise à déstabiliser l'ordre moral et social par la démonstration de la fragilité de son arbitraire. Cannibalisme, inceste, refus de sépulture sont autant de marques de l'affirmation d'un individualisme militant qui se donne 
pour tâche de proclamer l'immanence d'un monde débarrassé des dieux et de la transcendance. Rhétoricien pétomane se réclamant du chien, dormant en plein air, copulant en public et faisant l'apologie de la masturbation comme ascèse propédeutique à la maîtrise de soi, ${ }^{28}$ Diogène dit le refus d'une pensée prométhéenne, toute en ordre et en hiérarchie. $^{29}$

Pervers, à mi-chemin entre Diogène et Phryné, Lui est à la fois 'contre' et 'à côté' de la doxa; il la désigne et la reconnaît par le mouvement même qui la contourne et la désamorce. Mais le cynisme du pique-assiette Rameau est considérablement dégradé par rapport à l'intransigeance antique 'et solitaire' de Diogène. ${ }^{30}$ La cour Bertin, où il pérore jusqu'à son expulsion, n'est-elle pas une véritable pétaudière, réplique ellemême dégradée de la cité? (47). ${ }^{31}$ Certes, Lui agit comme principe cathartique, comme 'grain de levain qui fermente et qui restitue à chacun une portion de son individualité naturelle' (5). Cependant, si le Neveu est catalyseur de vérité, c'est presque malgré lui. Incurablement grégaire, ce parasite soucieux de confort, sinon de luxe, voit dans la satisfaction de ses besoins le suprême parangon. Face au philosophe, qui fait l'apologie de l'esprit de sacrifice et met un point d'honneur à se démarquer de la pantomime des gueux, en déclarant: 'Je veux mourir si cela [être comme Diogène] ne vaudrait pas mieux que de ramper, de s'avilir et se prostituer' (107), Lui reconnaît être 'un fainéant, un gourmand, un lâche, une âme de boue' (107). Il avoue être prêt à s'avilir pour '[...] un bon lit, une bonne table, un vêtement chaud en hiver; un vêtement frais, en été; du repos, de l'argent, et beaucoup d'autres choses [...]' (107). Derrière Diogène se profile Aristippe.

\section{L'ABJECT ET LE SUBLIME}

La figure de l'aveu organise ainsi dans le texte un réseau narcissique fondé sur l'abjection. L'abject n'est-il pas, en dernière instance, le signe d'une crise narcissique? Qu'est-ce qu'avouer pour le Neveu sinon jouir par l'autre et dans l'autre de l'image qu'il projette, fût-elle abjecte, et obtenir par là même la reconnaissance de son originalité, et donc de son identité? Lorsque le philosophe exprime son étonnement devant la componction du Neveu à exhiber ses défauts, celui-ci lui répond:

Je voyais plus à gagner qu'à perdre à vous avouer le reste [...] S'il importe d'être sublime en quelque genre, c'est surtout en mal [...] J'ai voulu que vous connussiez jusqu'où j'excellais dans mon art, vous arracher l'aveu que j'étais au moins original dans mon avilissement .... (72; nous soulignons) 
L'originalité du Neveu s'affirme par la prise en charge de sa propre désignation comme monstre. Le monstre n'est plus l'objet victimisé par la puissance nominative/monstrative de l'autre. Il triomphe dans la revendication de sa marginalité qui fonde l'exhaussement de l'originalité du sujet au-dessus de la médiocrité du commun. Paraphrasant De Quincey, nous pourrions intituler le discours de Lui: 'De la perversité considérée comme un des Beaux-Arts. ${ }^{32}$

Mais comment peut-on être sublime dans l'abjection? Depuis le père Rapin et son célèbre $D u$ grand et du sublime dans les moeurs et dans les différentes conditions des hommes, le sublime a en effet cessé de n'être attaché qu'aux seuls arts d'écrire et de parler: '[...] il peut y avoir du sublime en toutes choses. ${ }^{, 33}$ Il devient ainsi possible, par un détournement subversif de la doctrine classique, d'être sublime dans la perversité. A l'instar de De Quincey, qui considère le crime d'un point de vue esthétique et qualitatif, abstraction faite de toute considération morale, Lui apprécie toute action abjecte à la façon d'une œuvre plastique. Son narcissime abjectal déstabilise et scandalise le philosophe:

Je ne savais, moi, si je devais rester ou fuir, rire ou $m$ 'indigner [...] Je commençais à supporter avec peine la présence d'un homme qui discutait une action horrible, un exécrable forfait, comme un connaisseur en peinture ou en poésie examine les beautés d'un ouvrage de goût .... (76)

Outre l'exemple de Palissot, et surtout celui de Bouret, à qui le Neveu confère la première place dans son Panthéon pour son incarnation de 'la plus sublime idée' du génie dans la perversité (51), l'épisode du renégat d'Avignon nous montre un individu abject et pervers, non parce qu'il vole son riche ami israélite, mais parce qu'il condamne gratuitement à mort celui qu'il a volé et à qui, de surcroît, il est redevable de tant de bontés:

[...] le renégat se lève, dépouille le Juif de son portefeuille, de sa bourse et de ses bijoux; [...] Jusqu'ici, le renégat n'est qu' $[. .$.$] un coquin méprisable à qui personne$ ne voudrait ressembler. Le sublime de sa méchanceté, dit Lui admiratif, c'est d'avoir lui même été le délateur de son bon ami l'israélite, dont la sainte Inquisition s'empara à son réveil, et dont, quelques jours après, on fit un beau feu de joie. (75; nous soulignons)

La radicale altérité de cet 'acte gratuit' est irrécupérable par le discours de la doxa, laquelle est impuissante face à la 'cruauté cynique' du renégat, véritable modèle d'abjection. Cet élément est fondamental 
en ce qu'il renforce la filiation cynique du Neveu, qui nous offre par ailleurs un portrait du parasite en carnassier:

Des loups ne sont pas plus affamés; des tigres ne sont pas plus cruels. Nous dévorons comme des loups, lorsque la terre a été longtemps couverte de neige; nous déchirons comme des tigres tout ce qui réussit. (57)

Objet de mépris pour Moi, qui reconnaît l'intolérable en l'autre, Lui, qui revendique sa propre abjection, la reconnaît en lui-même, ce qui ne va cependant pas sans une certaine amertume qui explose sporadiquement. ${ }^{34}$ Ses deux attitudes constituent deux saisies du réel radicalement opposées. En rejetant l'abject hors de soi, le philosophe, malgré ses atermoiements et peut-être à cause de ses atermoiements, se pose comme entité artificiellement unifiée, ordonnée, conséquente, extérieure au phénomène 'Neveu de Rameau' qu'elle observe et étudie. Mais cette entité se fissure sous l'effet d'une contamination par l'abject.

La perversité du Neveu confronte le philosophe à sa propre abjection. Comparant sa femme, dont il se plaît à évoquer les charmes 'persuasifs', à 'une espèce de philosophe' (108), Lui nous entraîne vers une inévitable conclusion: le philosophe se prostitue. Le Socrate-'philosophe entremetteur' du Banquet de Xénophon est ici convoqué ${ }^{35} \mathrm{Nul}$ ne semble donc pouvoir échapper à l'abjection. Toutefois, si la découverte de l'abject en soi est incontournable pour le philosophe, l'acceptation de cette réalité lui est problématique.

$\mathrm{Au}$ contraire, la reconnaissance et l'aveu de son abjection s'accompagnent chez le Neveu d'un exhibitionnisme jubilatoire. Sa grande particularité ne réside pas dans la nature intrinsèquement monstrueuse de sa personnalité, mais dans la conscience abjectale de soi qu'il manifeste:

Il avouait les vices qu'il avait, que les autres ont; mais il n'était pas hypocrite. Il n'était ni plus ni moins abominable qu'eux; il était seulement plus franc, et plus conséquent; et quelquefois profond dans sa dépravation. (93)

Parce que l'abjection est en elle-même à la fois répulsion et fascination, condamnation et effusion, elle marque l'impossibilité fondamentale pour le sujet de se détacher totalement de ce qui le menace. En reconnaissant en lui-même la présence de l'abject, le Neveu se pose comme soi et l'autre à la fois et intègre sans les réduire les éléments qui le menacent comme sujet. D'où le malaise du philosophe et la clausule sybilline du morosophe Rameau: 'Rira bien qui rira le dernier' (109). Privilège du Neveu, le 'dernier' mot, qui n'est en fait que le dit de 
l'aporie, n'est pas sans évoquer la tirade où il énonce le renversement carnavalesque du sage et du fou, dans un cadre dont nous avons déjà, cependant, souligné les limites:

Il n'y a pas de meilleur rôle auprès des grands que celui de fou [...] Moi, je suis le fou de Bertin et de beaucoup d'autres, le vôtre peut-être dans ce moment, ou peut-être vous le mien: celui qui serait sage n'aurait point de fou; celui donc qui a un fou n'est pas sage; s'il n'est pas sage il est fou et peut-être, fût-il le roi, le fou de son fou. (61)

\section{DE L'INDIVIDU ${ }^{36}$}

Bouffon, morosophe, sublime coquin, le personnage du Neveu illustre symptomatiquement la montée dans le champ philosophique des Lumières du concept d'individu. Son credo discordant s'énonce en ces termes:

[...] ce sont des dissonances dans l'harmonie sociale qu'il faut savoir placer, préparer et sauver. Rien de si plat qu' une suite d'accords parfaits. Il faut quelque chose qui pique, qui sépare le faisceau, et qui en éparpille les rayons. $(94)^{37}$

De fait, Le Neveu de Rameau met en scène l'orthodoxie en crise du sujet-philosophe et l'hétérodoxie triomphante de l'individu-morosophe. A la persona figée de Moi, qui croit illusoirement pouvoir se tenir à l'écart de 'la vile pantomime' (107), le Neveu oppose l'évanescence de Protée et la dissémination d'Argus. Prototype de la sagesse morosophique, le Neveu affirme: 'Personne ne me connaît mieux que moi"(18), et déclare également connaître le 'mépris de soi' (22). Ce faisant, il désigne la reconnaissance de l'abject au-dedans-de-soi comme moment primordial de la constitution de l'identité du 'sujet', mais d'un sujet problématique, bien éloigné de l'hypothèse humaniste de la transparence à soi: 'Que le diable m'emporte si je sais au fond ce que je suis' (56). Le Neveu dit le savoir du non-savoir. Il revendique constamment son originalité et ne veut à aucun prix être objet ou victime sans son consentement. Il se pose comme personne désirante exerçant son libre arbitre et se distingue du contrat collectif dans un mouvement qui le désigne comme individu: 
Je veux bien être abject, mais je veux que ce soit sans contrainte. Je veux bien descendre de ma dignité [...] Chacun a la sienne; je veux bien oublier la mienne, mais à ma discrétion et non à l'ordre d'autrui. (46-47)

La répétition du syntagme 'je veux' signale l'importance de l'expression du libre-arbitre et de l'individualité chez le Neveu. ${ }^{38}$ Ainsi se dessinent deux trajets superposés: celui de l'abjectio et du dejectio, qui se caractérise par un mouvement d'expulsion/exclusion et de descente, et celui du subjectio, qui est au contraire élévation. Quelles conséquences pouvons-nous en tirer? Comment la revendication narcissique de l'abjection peut-elle participer d'un procès constitutif de soi? Comment ce qui est étymologiquement désigné comme le commun, le bas, peut-il constituer le 'fondement' de la revendication narcissique d'une originalité positivisée, positivisée parce que subjectivisée? Un tel discours, qui questionne les fondements ontologiques de la philosophie classique, marque bel et bien le passage, moment nécessairement mixte et ambigu, d'une philosophie du sujet à une philosophie de l'individu.

Dans son ouvrage L'Ere de l'individu, où il définit la philosophie du sujet comme valorisation humaniste de l'autonomie, i.e. comme rapport au monde dans lequel l'homme se pense comme fondement, Alain Renaut voit dans l'émergence de la monadologie leibnizienne le véritable acte de naissance d'une philosophie de l'individu. ${ }^{39}$ Par ce changement, Renaut voit s'instaurer chez les Modernes une différenciation entre l'humanisme, qui est valorisation de l'autonomie, et l'individualisme, qui est valorisation de l'indépendance. Dans cette perspective, chaque individu contribue alors, par la culture de son indépendance et la soumission à la loi de sa nature, à manifester l'harmonie et la rationalité de l'univers. Reconnaissant l'individu comme une figure du sujet, en ce que son affirmation présuppose 'l'installation de l'homme comme "valeur propre" dans un monde non intrinsèquement hiérarchisé,' Renaut souligne que

[...] ce que sape l'individualisme en ne retenant d'autre valeur que celle de l'affirmation du Moi, c'est paradoxalement (puisqu'il en procède) l'idée même d'autonomie. La perspective de soumission à des lois que je me suis moi-même données suppose en effet la possible référence à une telle ipséité du moi-même, posée comme distincte de ce qui, en moi, s'y soumet. L'idéal humaniste d'autonomie requiert donc en moi la définition d'une part d'humanité commune, irréductible à l'affirmation de ma seule singularité et à laquelle ma singularité doit se soumettre: tel est ce que, par définition, l'individualisme nie 
en posant qu'il n'existe que des différences irréductibles et que ce sont ces différences elles-mêmes qui doivent 'faire loi' et qui définissent la valeur. ${ }^{40}$

Pour définir le terme 'individu', les dictionnaires du XVIIème siècle -que ce soit le Richelet, le Furetière ou le Dictionnaire de l'Académie-reprennent l'acception scolastique 'd'être formant une unité distincte dans son espèce', tout en en déplaçant le sens de la métaphysique vers la science de la nature. Est également mentionné l'emploi péjoratif du terme: 'On dit aussi en raillerie d'un homme qui s'aime bien, lit-on dans Furetière, qu'il a bien soin de son petit individu. ${ }^{41}$

Ainsi émerge un type d'individu marqué par l'affirmation narcissique de sa forte subjectivité. Mais il faut attendre les articles 'Identité' et 'Individu' de l'Encyclopédie pour voir s'infléchir sensiblement le champ sémantique de ce terme, par une prédilection manifeste pour $l^{\prime}$ individu au détriment de l'espèce. ${ }^{42}$ Ce discours s'appuie sur la redéfinition lockienne du principe d'individuation, telle que développée dans Essay Concerning Human Understanding:

[the principium individuationis] determines a being of any sort to a particular time and place, incommunicable to two beings of the same kind [...] In the state of living creatures, their identity depends not on a mass of the same particles, but on something else. For in them the variation of great parcels of matter alters not the identity: an oak growing from a plant to a great tree, and then lopped, is still the same oak; and a colt grown up to a horse, sometimes fat, sometimes lean, is all the while the same horse: though, in both these cases, there may be a manifest change of the parts; so that truly they are not either of them the same masses of matter, though they be truly one of them the same oak, and the other the same horse. $^{43}$

Distinguant entre deux sortes d'identités, l'identité de substance, celle de l'atome, qui reste toujours la même, et l'identité de ressemblance, celle de l'être vivant, qui est quant à elle sujette à des vicissitudes mais garantie par la conscience et la mémoire, l'article 'Identité' de

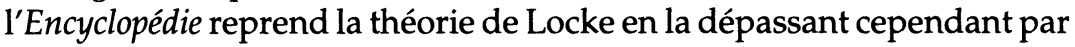
sa volonté de souligner la spécificité de cette problématique identitaire pour l'être humain:

Nous concevons différemment l'identité en différents êtres; nous trouvons une substance intelligente, toujours précisément la même, à raison de son unité ou indivisibilité, quelque modification qu'il y survienne, telles que ses pensées ou ses sentiments. Une âme n'en est pas moins précisément la même, pour éprouver 
des changements d'augmentation ou de diminution de pensées ou de sentiments; au lieu que dans les êtres corporels, une portion de matière n'est plus dite précisément la même, quand elle reçoit continuellement augmentation ou altération dans ses modifications, telles que sa figure et son mouvement. ${ }^{44}$

Débarrassé de l'harmonie préétablie leibnizienne, l'univers du Neveu, s'il retient un certain principe 'monadique' d'individuation, n'admet cependant que la matérialité d'une existence singulière posée comme seule réalité de référence possible:

Le point important, confie le Neveu, est que vous et moi soyons, et que nous soyons vous et moi; que tout aille d'ailleurs comme il pourra. Le meilleur ordre des choses, à mon avis, est celui où j'en devais être, et foin du plus parfait des mondes, si je n'en suis pas. (14)

Diderot abordera les rapports de l'Un et du multiple dans la question essentielle de l'identité à plusieurs reprises dans son œuvre, que ce soit dans le Discours de la poésie dramatique, le Salon de 1767 ou encore dans les Réflexions sur le livre De l'esprit par M. d'Helvétius. ${ }^{45}$ Mais à l'instar de la position encyclopédiste, c'est à cet être vivant particulier qu'est l'être humain que le philosophe s'intéressera.

Le néologisme 'individualité" ${ }^{\prime 46}$ n'apparaît que deux fois dans son œuvre, mais dans des contextes qui attestent l'intérêt du philosophe pour cette question: au début du Salon de $1767^{47}$ et dans le prologue du Neveu de Rameau (5), dont nous savons toute la valeur programmatique. L'individualité correspondrait ainsi chez Diderot à la nécessité de penser autrement le sujet, voire de penser un autre-du-sujet. Jamais peut-être la complexité du problème n'aura-t-elle été mieux exposée que dans Le Neveu de Rameau.

\section{RIRA BIEN QUI RIRA LE DERNIER}

Tantôt replet comme Silène, tantôt maigre comme Diogène (4), le Neveu est à la fois toujours différent et toujours le même: 'N'est-il pas vrai, dit-il au philosophe, que je suis toujours le même?' (109) Il s'agit pour Diderot de penser l'identité dans la métamorphose, l'individu dans ses avatars. Le philosophe, qui écrivait avoir cent physionomies différentes en un jour, ${ }^{48}$ élabore dans Le Neveu de Rameau une subtile pensée de l'hétérogène. Telle pensée suspend, par son mouvement, l'inaltérabilité du sujet autonome pour lui substituer le flottement sub- 
jectif de l'individu singulier. A l'image de la joute oratoire de Moi et de Lui qui demeure, jusqu'à la clausule emblématique, sous le signe de la non-résolution, la philosophie du monde de Diderot est non dialectique et consacre la vertu catalysatrice du stade des objections. Le personnage du Neveu procède ainsi d'une mise en question du statut du sujet dans la philosophie classique. Lui n'est plus cette instance souveraine capable de connaître, non seulement le monde, mais aussi se connaître ellemême et d'être à elle-même sa propre lumière: 'Je n'ai pensé de ma vie, déclare-t-il, ni avant de dire, ni en disant, ni après avoir dit' (56). Le sujet 'est agi' plus qu'il n'agit: il est une énigme, une 'boîte noire.' Prototype du savoir du non-savoir, Lui constitue une variété de l'irrationnel et porte une ombre au discours anthropologique -humaniste, dirait Renaut- des Lumières. Il refuse de s'élever à 'cette hauteur où tout se confond' (103). Ce qui lui importe, c'est son être-au-monde: 'Je suis dans ce monde et j'y reste [...] c'est toujours à l'appétit que j'en reviens, à la sensation qui m'est toujours présente [...]' (103). De son singulier discours, labile et contradictoire, émane une enthousiaste approbation narcissique et individualiste du monde. Hommage à la tradition rabelaisienne, le rire final de Rameau n'est peut-être rien d'autre que l'affirmation du pouvoir subversif du morosophe face aux Sorbonnagres de tous les temps.

Thierry Belleguic

Université Wilfrid Laurier

\section{Notes}

1 Lewinter, Roger, Diderot ou les mots de l'absence, Paris, Champ Libre, 1976: 9. Si cette réflexion recoupe nos préoccupations, il ne s'agira cependant pas dans ces pages de 'formalisme existentiel' ou de 'métaphysique analytique', pour reprendre les termes de Lewinter, qui examine la complexe dynamique du désir à l'oeuvre dans la création diderotienne. Notre démarche est d'ordre ontologique et phénoménologique. La question du recours à la psychanalyse, des limites de sa pertinence ainsi que des modalités de son application pour notre étude resterait à établir avec précision et nécessiterait une longue mise au point méthodologique et théorique, impossible ici. Précisons également d'emblée que le narcissisme que nous analyserons doit être entendu dans une acception qui émerge au XVIIème siècle et qui caractérise une conscience fortement subjectivisée qui choisit le soi comme objet privilégié d'attention.

2 'Né sous la (capricieuse) malice de tous les Vertumnes réunis...', trad. J. Fabre, Le Neveu de Rameau, éd. J. Fabre, Genève, Droz, 1950: 3. Toutes les citations du texte renverront à cette édition, dont nous avons cependant modernisé l'orthographe. Du fait de leur nombre important, elles apparaitront dans le corps du texte. 
3 Jauss, Hans Robert, 'Le Neveu de Rameau. Dialogique et dialectique ou Diderot lecteur de Socrate et Hegel lecteur de Diderot,' Revue de métaphysique et de morale, no 2,1984 : 145-81, 147.

4 Nous renvoyons à la réflexion de G. Gurvitch sur les 'dialectiques binaires', ainsi qu'à l'analyse herméneutique du problème que H. R. Jauss propose dans l'article cité, où il distingue et oppose 'les antithèses dialogiques de l'époque des Lumières avancée' et 'la dialectique monologique de l'Aufklärung achevée' (154).

5 Et ceci en dépit de la tradition du dialogue philosophique de Platon à Berkeley, dont $\mathrm{M}$. Bakhtine a signalé la structure foncièrement monologique dans sa Poétique de Dostoïevsky, (Paris, Seuil, 1963).

6 Starobinski, Jean, 'Diogène dans Le Neveu de Rameau', Stanford French Review, Fall, 1984, 147-67, 163-65; Jauss, op. cit., 147-49. Ménippe n'est-il d'ailleurs pas 'un des derniers cyniques de l'école ancienne', comme le rappelle Diderot dans l'article 'Cynique' de l'Encyclopédie? (Encyclopédie ou dictionnaire raisonné des sciences, des arts et des métiers, 17 vol., Paris, Briasson, David, Le Breton, Durand, 1755-74, t. IV: 598). Certes, c'est un modèle dégradé. Nous y reviendrons. Et Starobinski d'écrire en conclusion à son article: 'Les propos qui s'échangent dans la satire diderotienne ne trouvent leur patron légitime qu'en Ménippe, le disciple imparfait, l'écrivain spéculateur, sarcastique et incapable de résignation' (165). Jauss énumère les modèles ménippéens utilisés par Diderot: 'Parmi eux, on retiendra notamment: la situation exceptionnelle d'un homme 'placé sur un seuil', la liberté excentrique de 'l'invention philosophique', la mise à l'épreuve scandaleuse des 'ultimes vérités', l'expérimentation morale et psychologique allant jusqu'au dédoublement de la personnalité et, enfin, la transformation de l'identité humaine en un ensemble de rôles, cette transformation étant réalisée par une mise en scène du discours de l'autre (op. cit., 148). Jauss ne fait d'ailleurs en cela que résumer l'analyse bakhtinienne de la satire ménippée (op. cit., 151-69).

7 Jauss (op. cit., 146) reprend l'expression de Galle, dont il mentionne les travaux précurseurs. Voir Galle, Roland, 'Diderot oder die Dialogisierung der Aufklärung', Neues Handbuch der Literaturwissenschaft, vol.13, éd. J. von Stackelberg, Wiesbaden, 1980: 209-48.

8 Nous laissons délibérément de côté la question de l'Autre lacanien. Précisons également que l'autre-au-dedans-de-soi, suggestion de Max Vernet, est la seule formule qui nous ait semblé éviter l'ambiguïté de l'autre-en-soi, l'en-soi appartenant à une tradition philosophique à part entière.

9 Il ne peut s'agir ici que d'avatar. En effet, Le Neveu de Rameau est le lieu de la rencontre problématique de la tradition et de la modernité. Cf. Bernstein, Michael André, 'When the Carnival Turns Bitter: Preliminary Reflections Upon the Abject Hero, in Bakhtin: Essays and Dialogue on his Work, G. Saul Morson ed., Chicago, Chicago U.P., 1986, 99-121. Si le Neveu porte encore les attributs du fou (ubiquité, contact animal, rapport avec le règne des morts, même métaphorisé), le contrat carnavalesque n'en est pas moins rompu: 'Mais les temps ont changé: le fou de Bertin, en jouant ce jeu-là, court à sa perte. Car il inflige une blessure narcissique à l'enrichi qui le protège [...] Fou, flatteur, parasite: en ce domaine, comme en tous les autres, Rameau est un hybride. Il mêle en lui plusieurs fonctions archétypiques; mais il les mêle imparfaitement, inégalement. [...] L'échec du fou résulte de la fausseté d'une reprise du vieux rôle du bouffon, dans un monde où la contingence matérielle des sources du pouvoir est devenue pleinement apparente' (J. Starobinski, 'Le Diner chez Bertin', in Poetik und Hermeneutik, Das Komische, vol.7, München, Wilhem Fink Verlag, 1976:195-96). 
10 C'est dans cette acception précise que le narrateur de Jacques le fataliste interpelle le lecteur en ces termes: 'Avez-vous oublié que Jacques aimait à parler, et surtout à parler de lui, manie générale des gens de son état, manie qui les tire de leur abjection, qui les place dans la tribune, et qui les transforme tout à coup en personnages intéressants?' (nous soulignons). CEuvres complètes, Paris, Hermann, 1975-, t. XXIII: 189.

11 P. Gobin ('Transhistoricité des genres non-canoniques' in Renouvellements de la théorie et de l'histoire littéraire, éd. Eva Kushner, Ottawa, 1984, Actes du colloque international de 1982, Société Royale du Canada / Association internationale de littérature comparée, 53-63) et M. Lever (Le Sceptre et la marotte, Paris, Hachette, 1985, coll. Pluriel, 164-200) ont respectivement analysé la fonction dramatique et l'historique du morosophe. Le morosophe, c'est en effet le bouffon, le 'fou-sage', le 'sot-pas-si-sot' (Gobin, 56) qui peut, selon qu'il joue ou non la comédie, s'incarner en bouffon ou devenir philosophe sans le savoir.

12 Bakhtine, Mikhail, L'Oeuvre de François Rabelais et la culture populaire au Moyen Age et sous la Renaissance, Paris, Gallimard, 1970: 49.

$13 \mathrm{M}$. A. Bernstein voit dans le personnage du Neveu l'émergence de ce qu'il appelle le 'héros abject': 'Jean-François Rameau is, I believe, modern literature's first fully realized Abject Hero, the first self-conscious "wise fool" sufficiently versed in the traditional satires to understand the requirements of his role, precariously enough situated in his milieu to feel he has no other social position open to him, and embittered to the extent that he becomes unable as well as intermittently unwilling to fulfill these requirements. It is important, moreover, to stress that all of these attributes must be present for a character to be considered an Abject Hero' (op. cit., 112). En dépit du choix discutable du terme 'héros', le texte de Bronstein propose une stimulante analyse de l'inscription du Neveu de Rameau dans la tradition saturnale.

14 Kristeva, Julia, Pouvoirs de l'horreur, Paris, Seuil, Coll. Points, 1980: 8-39.

15 Cependant, si Diderot admet l'idée d'une sensibilité inhérente à la matière en général, il s'oppose à la réduction de toutes les fonctions intellectuelles à la sensibilité.

16 Kristeva, Julia, Histoires d'amour, Paris, Denoël, coll. Folio essais, 1983:153.

17 Nous renvoyons à la concordance établie dans Entretiens sur Le Neveu de Rameau (Duchet, Launay, [Paris, Nizet, 1967]: 287-402). Il y aurait tout un travail d'analyse de détail à faire sur la modalisation des discours respectifs de Moi, de Lui et du narrateur dans le champ sémantique de l'abjection.

18 Serres, Michel, Le Parasite, Paris, Grasset, 1980:190-91.

19 Dans la plupart des cas en effet, si l'on exclut peut-être certaines aversions alimentaires -encore cela reste-t-il à prouver- c'est originellement l'exclusion qui provoque l'abjection et non pas l'inverse.

20 Nous utilisons ce terme dans l'acception que lui donne C. Rosset dans La Logique $d u$ pire, (Paris, P.U.F., 1971). L'approbation serait l'acceptation du primat du hasard, la reconnaissance que la vie n'est que ce qu'elle peut être. Selon Rosset, l'approbation serait, avec son contraire qui est le suicide 'le seul acte dont la disponibilité soit laissée au sujet de l'action, à l'homme, 'c'est-à-dire comme la seule forme d'acte' (46). Rosset ajoute encore: 'L'acte dont il s'agit ne concerne que le mode selon lequel une personne se représente à elle-même ses pensées et ses actions, à chaque instant d'une existence dont aucun acte ni aucune représentation ne lui appartiennent en propre' (44). 
21 Kristeva, Pouvoirs de l'horreur, 12.

22 Nous renvoyons le lecteur à notre article 'Anthropologie diderotienne et cybernétique: une étude du parasitisme dans Le Neveu de Rameau', Recherches Sémiotiques/Semiotic Inquiries, vol. 11, no 1, 1991: 125-42.

23 En effet, s'il est maintenant 'un gros monsieur' qui a 'du foin dans [les] bottes' (29), le philosophe a lui aussi trotté 'sur le pavé' (id.), donnant des leçons de mathématique dont il ne savait mot (id.). Notre moral philosophe 'ne méprise pas les plaisirs des sens' et 'une partie de débauche, même un peu tumultueuse' (42), n'est pas pour lui déplaire. Ne va-t-il pas jusqu'à renchérir sur le raisonnement irrespectueux du Neveu, en soumettant le roi lui-même à la pantomime des gueux: 'Quiconque a besoin d'un autre, est indigent et prend une position. Le roi prend une position devant sa maîtresse et devant Dieu; il fait son pas de pantomime' (105).

24 'Il [Lui], écrit E. de Fontenay, se veut parasite qui signale et aggrave une crise qui grippe le système, 'fainéant, pervers, inutile', et non juste souffrant l'injustice sociale - 'perversion', Verkehrung, c'est en effet le mot que Hegel emploiera pour désigner le rapport du Neveu à la société de son temps' (Diderot ou le matérialisme enchanté, Paris, Grasset, 1981: 183). Notre lecture reste résolument en deçà d'une saisie de Lui en conscience déchirée opposée à la conscience simple du philosophe. Il est vrai que la lecture hégélienne du Neveu de Rameau nous en apprend beaucoup plus sur Hegel que sur Diderot. Cf. Hegel, G.W.F., $L a$ Phénoménologie de l'Esprit, 2 vol., trad. Jean Hyppolite, Paris, Aubier-Montaigne, éd.1966, vol. 2: 50-141.

25 Il ne s'agit cependant pas d'une prise de conscience totale qui consacrerait la toute-puissance introspective du Neveu, dont il ne faut surtout pas oublier la filiation morosophique. Dans la Lettre sur les sourds et muets, Diderot souligne d'ailleurs la difficulté, voire la gageure, que représente pour le sujet toute tentative $d$ 'introspection: 'Mais il en est de l'esprit comme de l'oeil; il ne se voit pas' (éd. Hermann, t.IV: 198).

26 Cette théorie est développée dans Le Rêve de d'Alembert où Bordeu déclare: 'On est heureusement ou malheureusement né; on est insensiblement entraîné par le torrent général qui conduit l'un à la gloire, l'autre à l'ignominie'. A la question de Mlle de Lespinasse: 'Et l'estime de soi, et la honte, et le remords? Bordeu répond: 'Puérilité fondée sur l'ignorance et la vanité d'un être qui s'impute à lui-même le mérite ou le démérite d'un instant nécessaire' (éd. Hermann, t. XVII: 186-87).

27 Kristeva, Pouvoirs de l'horreur, 23.

28 Ce point particulier de l'ethos cynique est symptomatiquement repris par Moi comme exemple révélateur du modèle d'indépendance diogénique (107). Ceci prouve bien, si besoin était, la nécessité de penser les rapports de Diderot aux protagonistes comme un 'partage des voix'. En d'autres termes, le Neveu n'est pas plus le porte-parole exclusif de Diderot que le philosophe Moi.

29 Dans son récent ouvrage Cynismes, Portrait du philosophe en chien, (Paris, Grasset, 1990), M. Onfray souligne l'importance de la pensée cynique quant à l'étude des rapports de l'individuel et du collectif: 'Le cynique s'efforce de produire une façon différente d'être au monde et subvertit la rhétorique classique qui invite à soumettre la singularité à la loi et aux principes de l'universel. Avec lui, l'antinomie entre l'individu et la société se résout au bénéfice du premier et, systématiquement, au détriment de l'instance normative sociale. Rebelle et solitaire, sa prestation est celle de la solitude pure' (67). 
30 Le cynisme antique, ou kunisme, ou encore cynisme kunique, qui est une 'propédeutique' à un savoir-jouir et fait partie d'une stratégie plus générale de libération de l'individu des réseaux coercitifs qui l'aliènent, doit être différencié du cynisme cynique ou cynisme moderne, qui est instrument d'aliénation, émanation d'un pouvoir 'machiavélique' qui l'utilise sciemment pour maintenir ses privilèges. Nous devons cette distinction à H. Niehues-Pröbsting (Der kynismus des Diogenes und der Begriff des Zynismus, München, Wilhem Fink verlag, 1979), distinction reprise par P. Sloterdijk (Kritik der zynischen Vernunft, Frankfurt am Main, Suhrkamp, 1983). En Lui coexistent une certaine positivité qui ressortit à la tradition kunique et une auto-réflexivité bordée d'insatisfaction et de ressentiment qui participe quant à elle du cynisme cynique. Diderot semble lui-même suggérer cette distinction dans l'article 'Cynisme' de l'Encyclopédie, lorsqu'il différencie Anthistène, Cratès et Diogène de Ménippe et de ses successeurs: 'Les faux cyniques furent une populace de brigands travestis en philosophes; et les cyniques anciens, de très honnêtes gens qui ne méritèrent qu'un reproche qu'on n'encourt pas communément: c'est d'avoir été des Enthousiastes de Vertu"(op. cit., t.IV: 599). Pour un approfondissement de la question du cynisme dans Le Neveu de Rameau, voir D. O'Gorman (Diderot the Satirist, Toronto, Toronto U.P., 1971: 143-44), J. Chouillet (La Formation des idées esthétiques de Diderot, Paris, Armand-Colin, 1973: 540-44) et J. Starobinski (op. cit., 1984:147-67).

31 Cf. le Dictionnaire de l'Académie de 1662: 'Terme de plaisanterie qui se dit d'une assemblée sans ordre, d'un lieu où chacun est le maître' (Cité par Fabre, op. cit., 277-78).

32 De Quincey, Thomas, On Murder considered as one of the Fine Arts, (1827-54), in The Collected Writings of T. De Quincey, 14 vol., vol. XIII, Edinburgh, Adam \& Charles Black, ed., 1890.

33 Rapin, René, S.J., Du grand et du sublime dans les moeurs et dans les différentes conditions des hommes, CEuvres, t. II, Amsterdam, éd. Estienne Roger, 1709: 441.

34 Lui: 'Le dieu est absent; je m'étais persuadé que j'avais du génie; au bout de ma ligne, je lis que je suis un sot, un sot, un sot' (98). 'Ah, monsieur le philosophe, la misère est une terrible chose. [...] Je ne sais si elle aiguise l'esprit du philosophe; mais elle refroidit diablement la tête du poète' (100).

35 Xénophon, Le Banquet, Paris, Les Belles Lettres, coll. Budé, éd.1961: 50.

36 Les remarques qui suivent ne sont que les prémisses d'une réflexion plus ample à paraître sur la notion d'individualité chez Diderot.

37 Cf. Moi: 'Je commençais à supporter avec peine la présence d'un homme qui discutait une action horrible [...] comme un moraliste ou un historien relève et fait éclater les circonstances d'une action héroïque' (76; nous soulignons).

38 Ce libre-arbitre doit cependant être évalué à la lumière du célèbre commentaire de Diderot dans les Eléments de physiologie: '[...] le Je veux n'est qu'un mot [...]' (éd. Hermann, t. XVII: 484).

39 Renaut, Alain, L'Ere de l'individu, Paris, Gallimard, 1989.

40 'L'Ere des monadologies', Magazine Littéraire, no 264, avril: 48-51: Voir aussi L'Ere de l'individu, 115-51.

41 Cité par Y. Sumi dans son ouvrage Le Neveu de Rameau, logiques et caprices du Jeu, Tokyo, France Tosho, 1975: 169-171. Notre réflexion sur le concept d'individualité au XVIIIème siècle s'est inspirée de la très intéressante analyse de Sumi, à laquelle nous renvoyons (167-336). 
42 Article 'Identité' (op. cit., t.VII: 494-95); article 'Individu' (id., 684). Comme le fait remarquer Sumi (op. cit., 174), le renvoi à l'article 'Identité' à la fin de l'article 'Individu' tend à confirmer la place prépondérante donnée au concept d'individu dans la réflexion des encyclopédistes sur l'identité.

43 Locke, John, Essay concerning Human Understanding, 2 vol., New-York, Dover Publications, éd. 1950, vol.2: 441-42. [Ce passage est d'ailleurs cité en traduction dans l'article 'Individu' de l'Encyclopédie.] Nous soulignons la structure stylistique de la phrase à laquelle fait écho le passage suivant du Neveu de Rameau:

'Quelquefois, il est maigre et hâve [...] Le mois suivant, il est gras et replet [...] Aujourd'hui, en linge sale [...] Demain, poudré, chaussé [...]' (4).

44 Op. cit., 494.

45 Nombreux en effet sont les passages dans l'oeuvre de Diderot qui évoquent cette question: ' $L$ 'homme diffère de lui-même en mille circonstances où il survient le plus léger dérangement dans l'organisation.' (Réflexions sur le livre De l'esprit par M. d'Helvétius, Hermann, t.IX: 308), ou encore: '[...] moi-même je ne suis jamais dans un instant ce que j'étais dans un autre' (Discours de la poésie dramatique, éd. Hermann, t. X: 424).

$46 C^{\prime}$ est en effet à L'Andrométrie de Boudier de Villemert que l'on doit la première occurrence du terme en 1753, terme repris par le biologiste Bonnet dans son Essai analytique sur les facultés de l'âme en 1760, ainsi que dans ses Considérations sur les corps organisés en 1762. (Voir Sumi, op. cit., 179).

47 Ed. Hermann, t. XVI: 64.

48 Salon de 1767, éd. Hermann, t.XVI: 82. 